\title{
Italique
}

Poésie italienne de la Renaissance

VIII | 2005

Varia

\section{Onomastica petrarchesca. Per Il canzoniere}

Paola Vecchi Galli

\section{(2) OpenEdition}

\section{Journals}

Edizione digitale

URL: http://journals.openedition.org/italique/114

DOI: 10.4000/italique. 114

ISSN: 1663-4438

\section{Editore}

Librairie Droz

\section{Edizione cartacea}

Data di pubblicazione: 1 juillet 2005

Paginazione: 27-44

ISBN: 2-600-01024-6

ISSN: 1423-3983

Notizia bibliografica digitale

Paola Vecchi Galli, « Onomastica petrarchesca. Per II canzoniere », Italique [Online], VIII | 2005, online dal 05 octobre 2009, consultato il 10 décembre 2020. URL : http://journals.openedition.org/italique/ 114 ; DOI : https://doi.org/10.4000/italique.114 


\section{Paola Vecchi Galli}

O N O M A S T I A P E T R A R C H ES C A.

P E R I L CANZONIERE 


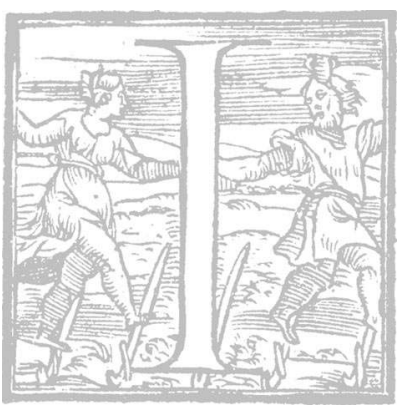

$L$ nome umiliante di Canzoniere $\grave{e}$ un'invenzione recente della critica petrarchesca: ${ }^{\mathrm{I}}$ un titolo vulgato ma bastardo, 'povero' ma fortunatissimo, che ha sbaragliato nel Novecento ogni altro concorrente antico e moderno (Rime, Sonetti e canzoni, Il Petrarca, ecc., e, prima ancora, l'originale, Rerum vulgarium fragmenta) per designare il libro di poesia di Petrarca. ${ }^{2}$ Sono numerosissime nell' ultimo secolo le edirioni petrarchesche che si sono chiamate Canzoniere: ${ }^{3}$ basti ricordare per tutte quella di Gianfranco Contini (Francesco Petrarca, Canzoniere, Testo critico e introduzione di Gianfranco Contini. Annotazioni di Daniele Ponchiroli, Torino, Einaudi, 1964). ${ }^{4} \mathrm{Ma}$ già ben prima di quella data il Canzoniere di Umberto Saba (Ig2I) aveva confermato un'attenzione al libro di rime che si nutriva anche dell'antecedente di Petrarca, portandone il nome.

Un po' diversa la prospettiva se, di là da Petrarca, poniamo il canzoniere nel solco degli studi sulla nostra letteratura delle origini e tre-cinquecentesca, italiana e romanza, come sostituto ed erede moderno degli antichi liber $e$ libellus, e dei romanzi libre, libel, libello (forse anche Petrarca ba definito la sua primissima raccolta di poesie volgari libellus). ${ }^{6}$ Anche qui il termine ha ottenuto ampio diritto di cittadinanza, a rappresentare il «libro di poesia lirica» di un solo autore, o la «raccolta organizzata di testi appartenenti ad autori diversis: ${ }^{7}$ in ogni caso un'opera di poesia composta o tramandata secondo una precisa intenzione strutturale, in tempi antichi o nei nostri giorni. ${ }^{8}$ In parte derivano da questo approccio teorico, in parte lo precedono, l'uso e l'abuso del titolo canzoniere applicato a ogni silloge di rime, anche la più estemporanea e posticcia, dai provenzali a Dante, dagli antichi (persino $i$ classici.) ai contemporanei, dall'Italia all'Europa: una specie di etichetta di comodo, dilagante fra Otto e Novecento, e oggi invece ricondotta a un impiego più consapevole e ortodosso. Lo si ricava ad esempio da un saggio memorabile di Avalle, I Canzonieri: definizione di genere e problemi di edizione, in La critica del testo. Problemi di metodo ed esperienze di lavoro, Atti del Convegno di Lecce (22-26 ottobre 1984), a c. di E. Malato, Roma, Salerno Ed., I985, pp. 363-82,9 ma anche dai più recenti Canzonieri della lirica italiana delle Origini, a c. di L. Leonardi (I. Il canzoniere Vaticano - Biblioteca Apostolica Vaticana, Vat. Lat. 3793; II. Il canzoniere Laurenziano - Biblioteca Medicea Laurenziana, Redi 9; III. Il canzoniere Palatino - Biblioteca Nazionale Centrale di Firenze, Banco Rari 217, ex Palatino 4I8; IV. Studi critici, Tavarnuzze-Firenze, SISMEL-Edizioni del Gallurzo, 2000). ${ }^{\text {Io }}$ 
Con varie sfumature di significato il canzoniere ha quindi preso piede come nome 'proprio' e come nome 'comune'. Per lo più dotato di un forte valore aggiunto, cioè dello statuto di genere - se non letterario - materiale, legato alle modalità di trasmissione delle nostre rime antiche. ${ }^{\mathrm{I}}$ Canzoniere si oppone ai componimenti spicciolati, veramente sparsi; presuppone organicità, sequenz̨e e strutture, strategia centripeta di un autore o di un antologista: in tutti i casi il suo archetipo, sottinteso o dichiarato, non può che essere il Canzoniere per eccellenza, quello di Petrarca.

Con queste premesse, il problema non è tanto di giustificare (o viceversa di deprimere) un nomen divenuto nel tempo titolo d'elezione dei Fragmenta un'intitolazione di immediata, incontrovertibile riconoscibilità - e categoria della critica, ma semmai di definirne la storia: risalire alle origini, per avvicinarci al momento e alle circostanze in cui la parola comincio ad avere cittadinanz̧a e identità nella tradizione della nostra poesia volgare.

Quanto a Petrarca, penso che si tratti di un nome, più che umiliante, umile, di una terminologia di servizio fatta circolare in origine da copisti e editori desiderosi di interpretare in qualche modo cio che la didascalia Francisci Petrarche laureati poete Rerum vulgarium fragmenta trasmetteva con chiarezza solo a pochi intendenti. Servirsene come di un nome comune (in altre parole, senza trascriverlo in corsivo $)^{\mathrm{I} 2}$ mi sembra un compromesso ragionevole, che riabilita la tradizione e salva capra e cavoli (l'intangibilità del titolo originale, da una parte, e dall'altra le credenziali storiche e critiche del titolo vulgato).

Altra minima distinzione potrebbe riguardare l'uso del canzoniere con o senza l'articolo determinativo. Il canzoniere si oppone a Canzoniere come il nome comune al nome proprio: il primo allude alla raccolta, sottintendendo che essa possa essere anche apocrifa, frutto delliniziativa di un editore moderno; mentre l'isolato 'Canzoniere', privo di articolo determinativo, dovrebbe piuttosto richiamare un'opera identificata da sempre come tale, il cui titolo è Canzoniere. ${ }^{\mathrm{I}}$

In realtà, quanto a quest'ultimo punto, non si dànno riprese antiche di Canzoniere come titolo di una raccolta di rime di un solo autore, in contesti diversi da quello petrarchesco. ${ }^{14}$ Questo silenzio è forse il segnale che quel nome, che pure veniva sporadicamente applicato a Petrarca, né risultava originale (e dunque classico, replicabile), né poteva rappresentare qualcosa di più di un'indicazione di genere.

Ma prima di arrivare alle conclusioni, bisogna ripartire da capo, vale a dire dall'uso moderno del termine, dalla sensibilità e dalla percezione, comune e generale, di canzoniere. Un titolo che si potrebbe definire, con Genette, rematico e descrittivo, ${ }^{15}$ che si confronta idealmente con il libro di Petrarca, e ne 
richiama la ratio strutturante. Se con il termine pensiamo a una scelta 'orientata' di poesie, una sorta di primogenitura spetterebbe al Guittone del 'Canzoniere' Laurenziano Rediano 9: ${ }^{16}$ a lui forse tocca la prima intuizione di una raccolta organica di rime d'autore, alla quale si sarebbero ispirati sia il Dante della Vita nova sia Petrarca. Dopo questo e altri studi, canzoniere non può più essere un vocabolo innocente: è invece un termine connotato con precisione (la 'forma-canzoniere', appunto), su cui si è sedimentata una ricca e importante letteratura critica.

Su un altro versante, parlare della rubrica dell'autografo petrarchesco, ms. Vaticano Latino 3195, come di un'intitolazione sibillina non è fuori luogo. Petrarca sanziona con quel titolo (Francisci petrarche laureati poete Rerum vulgarium fragmenta) la poetica del fragmentum lirico, che - lo ha insegnato Francisco Rico -, rinviando al tutto di cui $i$ singoli componimenti sono parte, indica nel contempo, inscindibilmente, la tessera e il mosaico che ne è composto. ${ }^{17}$ Fragmentum è parola poco usata da Petrarca, che, in tutta la sua opera, a quel che ho potuto vedere, solo in quattro occasioni ne sottolinea il valore simbolico e esistenziale. Oltre agli sparsa anime fragmenta di Secretum III 2I4, ci imbattiamo nelle cogitationum consumptarum fragmenta di Fam. XIII 6, 2, nei vite brevis fragmenta di Fam. XVII 5, Is e soprattutto in un brano di Fam. XXIV I2, 27 (la Responsio a Omero): «Reliquum est ut sparsa per totum epystole tue corpus querelarum tuarum fragmenta perstringam». In due casi su quattro $i$ fragmenta petrarcheschi sono sparsa: e confermano quella iunctura che, nell'idiografo Vaticano, verrà allusa e sciolta nella rubrica e nel primo verso del Canzoniere: Fragmenta // rime sparse. ${ }^{18}$

Nella stessa rubrica del Vaticano mi pare abbia un senso, e decisivo, anche l'accostamento fra l'appellativo che spetta all'autore (laureatus poeta) $e$ l'oggetto volgare dell'opera (rerum vulgarium fragmenta). Il poeta non ripudia le sue rime volgari, anzi, sintagmaticamente, avoca a sé il privilegio della laurea mentre intitola come cosa propria $i$ componimenti italiani. Un apparente ossimoro, posto alle soglie del libro, accoglie e sviluppa la distinzione/(con)fusione tra 'rimatore' e 'poeta' che Dante aveva collocato nel cuore della Vita nova: ${ }^{19}$

[...] prima è da intendere che anticamente non erano dicitori d'amore in lingua volgare, anzi erano dicitori d'amore certi poete in lingua latina [...]. E non è molto numero d'anni passati, che appariro prima questi poete volgari; ché dire per rima in volgare tanto è quanto dire per versi in latino, secondo alcuna proporzione (XXV). 
La distinzione fra poesia latina e volgare, ben nota a Petrarca, viene qui superata nei fatti: ${ }^{20}$ e non stupisce che, anche sotto questo aspetto, il titolo del ms. Vaticano potesse essere per $i$ lettori del tempo di difficile comprensione. Non basta. Che il fragmentum fosse parola chiave del suo universo semantico e concettuale erano in grado di saperlo Petrarca e i suoi lettori più attenti. Per di più, era solo la seconda volta che una raccolta di poesie italiane veniva denominata dall'autore: dando per scontato che il titolo del Vaticano Latino 3195 sia in tutto e per tutto originale (idiografo, quindi, anche se non autografo). ${ }^{21}$ Il primato - lo ba ricordato di recente Guglielmo Gorni - ${ }^{22}$ spetta d'altronde a un altro 'nome' petrarchesco (però non di mano di Petrarca), Fragmentorum liber, che Boccaccio pone in testa alla sua trascrizione della forma Chigiana del Canzoniere di Petrarca (ms. Chigiano L.V.I76). ${ }^{23}$ E la perfetta coincidenza, nelle due rubriche, del vocabolo più saliente e connotato (fragmentum) fa pensare che appunto di nome d'autore in entrambi $i$ casi si tratti.

Il titolo Fragmenta ha poi avuto anch'esso, nella tradizione letteraria del Quattrocento, una sua piccola fortuna: ad esempio, nei Fragmenta vulgaria del Suardi; ${ }^{24}$ nel Fragmentario poetico Isoldiano (Bologna, Biblioteca Universitaria, ms. I739); ${ }^{25}$ nell'intitolazione del medesimo manoscritto che apre le rime di Giovanni Testa Cillenio ("Domini Johannis Cyllenij viri eloquentissimi quedam fragmenta incipiunts); nel canzoniere per Angiola di Antonio Cornazano (I4I, IO-II: "Fra dolci appassionati miei fragmenti / che d'una Angiola viva ordino adesso»); nei Rerum vulgarium fragmenta di Nicolò Lelio Cosmico (conservati nel ms. Marc. It. IX.III) ${ }^{26}{ }^{2}$ in Tebaldeo (Rime, II9, 9-I0: «Fragmenti / che già per Flavia scrissi suspirando»); e, prima ancora, in una prosa di Domenico da Prato, che, fraintendendone in pieno il significato nel contesto petrarchesco, cosi riprende quanti

senza vergogna, presuntuosamente ardiscono di dire che li romanzi del famoso messer Francesco Petrarca sono fragmenta, cioè sono rimasugli e cose minuzzate e quasi proiecibili; ${ }^{27}$

a conferma di un'attenzione a Petrarca che, in casi come questi, non può che essere alimentata da una conoscenza diretta, anche se non sempre centrata, del ms. Vat. Lat. 3195. Come canzoniere, cosi frammento $e$ frammentario oscillano a loro volta tra un valore forte, epigonico, che riprende con piena consapevolezza il titolo di Petrarca (clamoroso l'esempio dei Rerum vulgarium fragmenta di Cosmico), e un uso più debole e meno connotato.

Non è del tutto vero, dunque, che Rerum vulgarium fragmenta sia rimasto a lungo un titolo per pochi, un'esclusiva dell' ambiente veneto che era custode materiale del manoscritto. Anche l'elenco delle testimonianze si va 
allungando: ai tre codici segnalati da Nadia Cannata (Bodleian Library, Montague d 32; Montpellier, Bibliothèque de la Fac. de Medicine, I980; Modena, Biblioteca Estense, It. 262$)^{28}$ se ne possono subito aggiungere alcuni altri: i mss. M 502 della Pierpont Morgan Library di New York, e Rediano II8 della Biblioteca Medicea Laurenziana di Firenze (scoperta recente di Maddalena Signorini); e i mss. Laur. XLI inf. 2 (Francisci Petra $<\mathrm{r}>$ che laureati Rerum vulgarium fragmenta) e Rediano I84 (da me reperiti durante $i$ lavori di schedatura per il Catalogo dei codici petrarcheschi fiorentini). Se il Rediano II 8 è di origine veneziana (è stato infatti trascritto da Leonardo Giustinian) e il ms. M 502 di provenienza viscontea, ${ }^{30}$ gli altri due manoscritti, entrambi toscani, provano come il titolo fosse noto anche fuori dagli ambienti dove si conservava l'originale dei Fragmenta. ${ }^{31}$ Ha una diffusione più ristretta, invece, la tradizione degli incunaboli, dall'edizione padovana Valderoco del $1472^{32}$ ad altre stampe che si dichiarano, come quella, ex originali libro extractae. Il Repertorium Bibliographicum di Hain è illuminante: contengono il titolo d'autore (di solito nella formula Francisci Petrarcae poetae excellentissimi rerum vulgarium fragmenta) anche l'explicit dell'edizione di Leonhard Achates (Venezia, I474: Hain I2759, IGI 7522); di Domenico Siliprandi (Venezia, I477: Hain 12766, IGI 7525, BMC V, 263); di Filippo di Pietro (Venezia, I482: Hain I275I e I2761, IGI 7526).

L'apparizione in più esemplari e in ordine che oggi appare sparso (vale a dire non prodotto da una strategia congiuntiva della tradizione) del nome voluto da Petrarca per il suo libro di rime - a maggior ragione se si tratta di copie lontane, anche nei contenuti, dall'originale Vaticano - ${ }^{33}$ non è un elemento da prendere sottogamba. Di certo non poligenetico, segnala invece un'indubbia contiguità con gli autografi padovani. Ma sottolinea anche la difficoltà dei copisti a intendere la rubrica del ms. Vaticano come parte inscindibile dell'opera: come paratesto, insomma, soglia ideologica del Canzoniere; e non, secondo la tradizione delle nostre rime antiche, come didascalia, fattore librario modificabile, secondo le necessità, di copia in copia. E se la sua presenz̧a costituisce un indizio importante, la sua assenza non significa, automaticamente, distanza o ignoranza dell'originale. Molti possono essere stati i motivi del silenzio. Il primo, e forse il più importante, è bibliologico: il manoscritto, a differenza del libro a stampa, non richiede, dato il circuito ristretto produttoreconsumatore che gli è sotteso, di anticipare col titolo il contenuto dell'opera. ${ }^{34}$ Ma vorrei pensare anche a una ragione diversa. Il titolo, quel titolo, non era chiaro a tutti; alcuni, gli amanuensi più fedeli o acuti, lo ricopiavano; altri no (e fra le eccezioni illustri possiamo contare il ms. Laurenziano XLI. Io, posseduto da Coluccio Salutati, e il Laurenziano XLI. I7, testimone precocissimo e principale della cosiddetta 'forma Malatesta' del Canzoniere). ${ }^{35}$ 
Prima di quei Fragmenta le rime, o la raccolta di rime, non avevano nome. Avevano già un titolo, invece, le opere volgari di Dante, dalla Vita nova al Convivio alla Commedia (anche se nulla oggi sappiamo della forma materiale degli autografi danteschi): un antefatto forse decisivo per Petrarca, su cui pure avrà agito, per il Canzoniere, la molla di una strategia strutturante in lui potentissima - di cui il titolo è elemento formativo e simbolico -, che, sotto la sorveglianza dell'autore, trasforma la somma di componimenti spicciolati in sequenza e in libro. ${ }^{36}$

Con l'eccezione di Dante (per la Vita nova) e di Petrarca non si dànno, sino a Quattrocento avanzato, intitolazioni specifiche del libro di poesia d'autore (che può venire rubricato genericamente come Rime o altro). Semmai, il nome (o, meglio, il titulus) tocca al manoscritto miscellaneo che le contiene, ${ }^{37}$ o $\grave{e}$ incluso nella didascalia che accompagna $i$ componimenti: come designazione dell'autore, dei suoi eventuali corrispondenti o della qualità metrica delle rime. La tradizione dei titoli (ma sarebbe più corretto parlare di 'intitolazioni', rubriche e didascalie: del peritesto, insomma) dei miscellanei e delle raccolte poetiche tre-quattrocentesche è del resto un capitolo della nostra storia letteraria ancora tutto da scrivere. Solo all'epoca della fioritura tardo-quattrocentesca e primo-cinquecentesca della poesia cortigiana, le cose cambiano: ${ }^{38}$ quanto al titolo, il vero discrimine è in quella zona d'ombra della tradizione della poesia in volgare che sta fra il manoscritto e la tipografia. Nella stampa, intestazioni e didascalie lasciano via via il posto ai titoli in frontespizio: un cammino che, in totale autonomia e con grande anticipo, Petrarca aveva per parte sua posto in essere con $i$ Rerum vulgarium fragmenta, anch'essi 'trasumanati' dall'occasione estemporanea e cortigiana (ancora testimoniata dalle rubriche del ms. Vat. Lat. 3196) e (ri) condotti alla spoglia, trascendentale solitudine della raccolta lirica. Molto poco ci si è interrogati sui motivi che portano Petrarca a rinunciare, nel Canzoniere, a ogni didascalia, vale a dire al colloquio diretto con la contemporaneità e con i posteri. Azzardo un'ipotesi: la ragione di questo silenzio potrebbe essere ricercata nel libro di rime degli autori latini, che gli giungeva composto di soli versi, spoglio di una qualsiasi prosa di accompagnamento. Anche il poeta moderno si affranca allora da ogni riferimento al suo presente; rinuncia a postille e a dediche (affidate se mai alle epistole, come la Var. 9 a Pandolfo Malatesta) perché soggette all'assalto e all'arbitrio della tradizione di copia; compone, all'antica, un libro di rime con un solo titolo d'autore, quello iniziale.

Per venire finalmente a nuovi esempi e applicazioni di Canzoniere in ambito petrarchesco, devo premettere che - dato interessante - non è soltanto alle stampe che si deve guardare, ma anche alla tradizione manoscritta del Quattrocento. Il nome è diffuso, se non a macchia d'olio, più di quanto non 
si sia percepito finora: ed $\grave{e}$, per quel che sappiamo, testimoniato dal XV secolo.

Nel significato di raccolta di rime di diversi autori, non si risale, in Italia, oltre il pieno Quattrocento, con il ms. Par. It. 1035, il Cansonero del Conte di Popoli (I468). ${ }^{39}$ Per Petrarca il censimento registra qualche testimonianza in piu: si va, in ordine decrescente, ${ }^{40}$ dal IsI6 - l'edizione bolognese di Tommaso Sclaricino Gammaro, Canzoniere et Triomphi, In Bologna, per maestro Francesco da Bologna, I5I6, BIBLLA 3564 (a cui si deve far seguire un'edizione di cui finora si è tenuto assai poco conto: Canzoniere et Triomphi, In Florentia, per Philippo di Giunta, III5, BIBLLA 3560) --41 al I484 - l'edizione Piero de Piasi con il commento di Girolamo Squarzafico (Finis dil canzionero di Franciescho Petrarcha per maistro Piero cremoneso dicto veroneso impresso in Vanesia adì i 8 del meso de augusto Mccclxxxiiii) - 42 $^{42}$ al I470 circa (I469-I474) - una postilla del ms. Silvestriano I89 dell'Accademia dei Concordi di Rovigo, c.54r (Seguitano alcune degne auctorita extracte delli comenti sopra elcanzonero del petrarcha compillati per lo eximo homo philelpho).$-^{43}$ Altri codici, di qualche anno più antichi, sono il ms. D'Orville sI4 della Bodleian Library (I450-I460 circa) e il ms. 392 della Bibliothèque Inguimbertine di Carpentras (I470 circa), che applicano entrambi a Petrarca il nome canzoniere: dunque ben documentato «fra la metà e il terzo quarto del Quattrocentos. ${ }^{44}$ Ancora più remote sarebbero un'indicazione contenuta nel commento dello Ps. Antonio da Tempo (I436?, ma a stampa solo nel I477, Venezia, Domenico Siliprandi), che definisce l'opera in versi del celeberrimo poeta canzonero, ${ }^{45}$ e la testimonianza della Vita di Petrarca di Luigi Peruzzi (I440 circa), che cosi riflette, in un passo che a me sembra particolarmente eloquente, sull'amore di Petrarca per Laura:

La quale [giovane] di bellezza, contanenza e costumi fu tale, quale lui cantando e scrivendo pone, chiamandola Laura per non pigliare il nome diminutivo, e anzi per sonarla e chiudere megli in versi, come si dimostra per una opera chiamata Canzionero, ne la quale sono inchiusi canzoni, sonetti e triumfi in stile di rimati versi, tanto leggiadri, gientili e limati, ch'è una maraviglia, conducendo tanto il suo dire al proposito di lei e di suo innamoramento [mio il corsivo]. ${ }^{46}$

A sentire Peruzzi, quindi, per canzionero si intende - agli albori stessi dell'uso del vocabolo - tutta l'opera volgare di Petrarca. Sono didascalie come queste che mi rendono perplessa: esito percio a condividere il giudizio secondo cui «il termine 'canzoniere' è sicuramente nome proprio, specifico della raccolta di Petrarcas. ${ }^{47}$ Né mi sembra che lo dimostrino con certezza altri esempi (come la rubrica del ms. di Carpentras che recita: «Incomincia la cantilena di meser Francesco Petrarca famosissimo poeta fiorentino chiamato il canzo- 
niere composto ad laude di Madonna Laura»). ${ }^{48}$ É invece probabile che $i$ 'titolisti' stiano cercando di risolvere, ciascuno per conto proprio, una difficoltà di fondo: vale a dire quella di sciogliere, di interpretare il nome originale dei Fragmenta - se pure erano in grado di attingervi -; di crearne uno nuovo, se $i$ componimenti erano anepigrafi; e, ammesso che fosse loro ben chiara la distinzione fra le diverse opere di Petrarca, di aggregare a essi anche altre rime di Petrarca e $i$ Triumphi.

Ma quanto e quando il nome Canzoniere si sia diffuso a designare l'opera volgare (non sempre i soli Fragmenta) di Petrarca resta incerto. Un punto fermo è intanto che, dove venga usato il nome Canzoniere (per una o più opere di Petrarca), il copista o l'editore aggiungono di norma altre definizioni, $o$ assegnano quel titolo a un organismo testuale che può comprendere $i$ Fragmenta $e$ il loro commento, o $i$ Fragmenta $e i$ Triumphi. In fondo, a questa regola non fa eccezione neppure il ms. Silvestriano 289 di Rovigo, che attribuisce il titolo a un estratto del commento di Filelfo al Canzoniere.

Su questa linea stanno tre nuovi manoscritti, emersi dai lavori di allestimento del Catalogo dei codici petrarcheschi di Firenze. ${ }^{49}$ Partiamo dal più recente, il ms. Rediano I84, imponente miscellaneo (con più di novecento componimenti) allestito a Firenze nella seconda metà del XV secolo (le date 1436 di c. 26v, 1464 di c. I50v e 1468 di c. I56r, che risalgono a due mani differenti, sembrano riferirsi all'epoca di composizione delle opere, non della compilazione del manoscritto)..$^{50} E$ poiché la mano principale, quella del fiorentino Baroncino di Giovanni Baroncini, sottoscrive fra il 1476 e il 1483 altri mss., come i Riccardiani 1330, 1376, $2580^{51}$ e il Magl. XXXV IOI, siamo orientati fra gli ultimi anni Settanta e i primi Ottanta del Quattrocento.

In questo codice la designaz̧ione di Canzoniere non è la sola di cui si fregia il libro. Non è una novità, e si può spiegare con l'incertezza onomastica, ma ancor più strutturale, che caratterizza la tradizione dei Fragmenta. Il copista principale del Rediano riporta il titolo originale dell'opera nella rubrica di c. 44r: "Illustris domini francisci petrarce de Florentia Rerum vulgarium fragmenta». Se della didascalia dell'idiografo sopravvive il nomen, è invece venuto meno il poeta laureatus, mentre Petrarca, secondo una strategia assai praticata nel Quattrocento, entra a far parte a pieno titolo del pantheon delle glorie fiorentine. ${ }^{2}$ Si è già detto come il 'nome' Rerum vulgarium fragmenta dia da pensare. Da dove Baroncino, e altri come lui, l'avessero ricavato non sappiamo: ma si è visto come a quest'epoca già circolasse l'edizione Valdezoco (I472) del Canzoniere, che riproduceva, direttamente dall'originale, il titolo voluto dall'autore. Non basta: il ms. Rediano I84 tramanda, a c. $48 \mathrm{r}$ e della seconda mano, l'avvertenza: «Segue la canzon dimesser francesco petrarcha $n^{\circ} 3^{8} /$ Ma nel chanzoniere traIsonetti e la 
$38^{a} \gg .{ }^{53}$ Il titolo canzoniere si affaccia quindi, anche se non da una posizione eminente, accanto al nome originale e ad altre formule più correnti (come a c. 9ov, ancora della mano principale: «Segueno anchora Canzoni e sonetti di messer Franciescho petraccha», dove è registrata la variante non umanistica, ma fiorentina, del cognome di Petrarca). ${ }^{54}$

Veniamo ora alla testimonianza più antica delle tre: quella del ms. XLI. I della Biblioteca Medicea Laurenziana (il cui copista si sottoscrive a c. I8 3 r: "Iacobus Macarius Venetus scripsit in civitate Senarum I463»). ${ }^{55}$ Questo il colophon, a c. igor del codice:

Qui finiscono el Canzonieri e' triumphi facti per lo elegantissimo e clarissimo poeta Messer francesco petrarca fiorentino che sono sonetti 3 ig e canzone 29 morale e Io madriale et 9 sestine et 6 triumphi in I 2 capitoli, etiam la vita sua finisce. Laus omnipotenti [mio il corsivo].

Un copista veneto impiega, a Siena, il nome Canzonieri, distinguendo senza ombra di dubbio fra le due opere in volgare di Petrarca (el Canzonieri e' triumphi). E un elemento su cui riflettere che $i$ Fragmenta siano definiti opus nell'incipit di c. Ir ("Poetae clarissimi FRANCISCI Petrarchae florentini OPUS HIC INCIPIT LEGE FOELICITER»), con chiara percezione che quella sequenza di rime è e va intesa come un libro.

Un po' diversa, infine, ma più eloquente, l'ultima testimonianza, quella del ms. Laurenziano XCI. inf. $2:^{56}$

Mcccclxvi // Questo libro si è di Fruosino di Lodovicho di Ciecie da Pragnio / et di sua mano et scritto del mese di febbraio et di marzo I $466 /$ e tratta di tutte le chanzone et sonetti e ballate e madriale / del famosissimo poeta messer Franciescho Petrarcha poeta / fiorentino et choxì anchora ci è scritto tutta la vita sua / e detto libro si chiama et è titolato Canzoniere di messer Franciescho [mio il corsivo].

Così, a c. Ir il copista firma e data (al I467 secondo lo stile fiorentino) $i$ Fragmenta: «detto libro si chiama et è titolato Canzoniere di messer Franciescho». Nomen e titulus dell'opera coincidono, e il trascrittore annota scrupolosamente la sua trouvaille onomastica, applicando la distinzione descritta limpidamente da Michele Feo: «nomen è il flatus vocis con cui si individua uno scritto, titulus è un elemento di tecnica libraria, la rubrica». ${ }^{57}$ In questo caso, il libro (l'unità strutturale e materiale composta di opere diverse) si chiama nel suo complesso Canzoniere; e quello stesso nomen deriva dal titulus, cioè dall'intestazione dell'opera.

Non basta. Oltre alla nota di possesso, a c. Ir-IIr la tavola alfabetica del manoscritto recita: "Chomincia le rupliche del libro titolato Chan/zoniere 
chompilato pello famosissimo poeta/messer Franciescho Petrarcha poeta fiorentinos. Ma nell'indice delle rupliche sono compresi tanto $i$ capoversi dei Rvf quanto quelli dei Triumphi, collocati in coda ai Fragmenta, di cui proseguono la numerazione. Ciò significa che il copista è ben in grado di distinguere fra le due opere, come testimoniano, per parte loro, altre due didascalie, collocate a mo' di colophon rispettivamente a c. I93v (Expliciunt sonitia cantilene madrialya / atque ballate excellentis poete / domini Francisci Petrarce) e a c. $234 \mathrm{v}$ (Finity i Triunfi chompilati per / messer Francescho Petrarcha poeta clarissimo). Dunque, insieme, rime e Triunfi compongono detto libro, il quale si chiama ed è titolato canzoniere di messer Franciescho. E del libro fanno parte, come negli antichi manoscritti trobadorici, anche un minimo corredo illustrativo - il ritratto dell'autore, qui a pagina piena a c. II' / v - e la sua Vita (nel testo di Leonardo Bruni): non componenti marginali, ma elementi di coesione di un insieme materialmente costituito da quel codice.

Questa testimonianza, unica nella sua puntualità terminologica, è forse di qualche aiuto per definire il passaggio del canzoniere da nome comune a nome proprio. Mi sembra decisivo, qui e in altri casi (come nella Vita di Luigi Peruzzi, ad esempio), che il vocabolo rimandi al 'libro' di Petrarca nel suo complesso, a un'unità strutturale e materiale di cui $i$ Fragmenta sono uno, ma non il solo, dei componenti. Forse qui - ammesso che il nome dipenda da quello che designava i miscellanei poetici, già testimoniato tra la Francia e la Spagna, e poi nell'Italia aragonese $-{ }^{58}$ potrebbe essere l'origine del Canzoniere di Petrarca: in uno sforzo interpretativo della tradizione che, fraintendendo l'unità voluta dall'autore per il libro di rime denominato Rerum vulgarium fragmenta, ha riconosciuto a tale unità solo un significato materiale, l'ha applicata a una pluralità di segni, iconici e letterari, del manoscritto e ha intitolato questa pluralità canzoniere. Ma se pure il termine è stato impiegato per designare tutta l'opera in versi di Petrarca, compresi $i$ commenti e $i$ Triumphi, ben presto però, e per iniziativa (non sappiamo quanto poligenetica) di copisti, commentatori e stampatori collocati fra il Nord e la Toscana, è poi passato a indicare anche $i$ soli Fragmenta. La ricerca di un nome per quei rimasugli e cose minuzzate e quasi proiecibili può aver condotto a questa soluzione d'emergenza: oggi come allora, a un scelta di comodo degli interpreti. Il manoscritto di Fruosino di Lodovicho di Ciecie da Pragnio è un anello importante di questa catena: non precursore, ma testimone di una tensione onomastica che vuole, fra Tre e Quattrocento, dare un titolo al Petrarca volgare denominando i libri in cui esso di volta in volta si incarna, le sue copie.

Come si vede, la questione non è solo terminologica, ma sostanziale: abbraccia cioè la consapevolezza dei Fragmenta di Petrarca come di un organismo a cui 
spetta un nomen che tutto lo rappresenti. Ho quindi forti dubbi che il termine canzoniere sia stato inteso, fin dalle origini, come nome proprio del libro di rime di Petrarca (un'alternativa al titolo voluto dall'autore): se non altro perché la tradizione della poesia volgare non rendeva necessaria questa presenza. Mi sembra invece possibile che, almeno inizialmente, l'uso di canzoniere per rappresentare rime, Triumphi, commenti e vite di Petrarca rinvii al significato collettivo, ossia comune, del nome (cioè indichi materialmente e genericamente, non specificamente, un libro composto da diverse opere di e su Petrarca). La percezione del termine è oscillante: se pure designa le sole rime di Petrarca, quel titolo guarda alla pluralità dei componimenti, sparsi e tuttavia tramandati, per ragioni che molti copisti ancora non sapevano né potevano capire a fondo, in gran numero e in forma di libro. Penso quindi a un compromesso della tradizione, a un'intitolazione che, con la parola che offriva il mercato, ha cercato di dare organicità e nome a un corpus che solo pochissimi lettori identificavano come tale (ricordiamo lo sconcerto di Domenico da Prato di fronte all'uso, a suo dire perverso e mistificante, del termine Fragmenta).

Restano però in ombra altri passaggi. Di certo il termine canzoniere, in antico, sostituisce solo sporadicamente altri titoli petrarcheschi più vulgati, come Canzoni e sonetti (quanto esso sia diffuso lo potrà dire solo il censimento completo delle copie del Petrarca volgare). Di certo quel nome accompagna, spesso e volentieri, altre intitolazioni del libro di rime di Petrarca; e allude a una coesione 'materiale' (più che a un'unità strutturale e simbolica) di cui $i$ Fragmenta sono $i$ principali, ma quasi mai gli unici componenti. Ma se è vero che, in un'accezione più ampia, il vocabolo è attestato in Italia dalla metà del XV secolo e in ambito aragonese, viene da chiedersi quale possa essere stata la trafila che lo ha portato molto presto (ricordiamo la Vita di Luigi Peruzzi, I440 circa) sino a Petrarca.

Quel che oggi sappiamo non è dunque ancora sufficiente per sciogliere l'enigma: dal canzoneri/canterino di Rosa fresca aulentissima al librochansonnier francese del $1328^{59}$ al cansonero del Conte di Popoli al Canzoniere di Petrarca il passo è lungo. Petrarca ha attraversato il cammino di questo nome e ne è stato segnato: il canzoniere/Canzoniere appartiene per sempre, umilmente, alla storia dei Rerum vulgarium fragmenta.

Paola Vecchi Galli 


\section{Paola Vecchi Galli}

I. La definizione di «nome umiliante di Canzoniere» per i Rerum vulgarium fragmenta spetta a Michele Feo, nella sua 'voce' Petrarca di Orazio. Enciclopedia oraziana, Roma, Istituto della Enciclopedia Italiana, I998, III, pp. 405-25, a p. 42 I. In apertura segnalo il mio debito nei confronti di N. Cannata, Dal "ritmo" al "canzoniere": note sull'origine e l'uso in Italia della terminologia relativa alle raccolte poetiche in volgare (secc. XIII-XX), in "Critica del testo", IV/2 (200I), pp. 397-429, che ha fornito coordinate storiche e documentarie importanti riguardo all'onomastica del libro di rime petrarchesco e del nome Canzoniere. Altra bibliografia sul tema è distribuita nelle note che seguono.

2. Il demerito maggiore, e insieme la fortuna del nome Canzoniere, è quello di avere soppiantato nella percezione dei lettori comuni il titolo originale dei Fragmenta di Petrarca. Per un aggiornamento della questione canzoniere, terminologica e storica, cfr. G. Gorni, Il canzoniere (1984), poi in Id., Metrica e analisi letteraria, Bologna, Il Mulino, I993, Pp. I I 3-34; N. Cannata, La percezione del Canzoniere come opera unitaria fino al Cinquecento, in L'io lirico: Francesco Petrarca. Radiografia dei Rerum vulgarium fragmenta, a c. di G. Desideri, A. Landolfi, S. Marinetti, in «Critica del testo», VI/I (2003), pp. I 55-76; Ead., Dal "ritmo" al "canzoniere" cit.; M. Signorini, Fortuna del "modello-libro" Canzoniere, in L'io lirico: Francesco Petrarca cit., pp. 133-54. Un'ultima avvertenza. Cerco di conservare in questa sede, e fin dove è possibile, la distinzione fra titulus e nomen, così come la propone Michele Feo, Fili petrarcheschi, in «Rinascimento», n.s., XIX (1989), pp. 3-89: è quasi superfluo però avvertire che nome è oggi comunemente soppiantato da titolo per indicare «il nome del libro, la definizione sintetica che serve a identificare un'opera» (così Il paratesto, a c. di C. Demaria e R. Fedriga, Milano, Edizione Sylvestre Bonnard, 200I, p. I 84).

3. In realtà, il nome Canz̧oniere diventa sempre più comune già a partire dalla prima metà dell'Ottocento. Lo nota Cannata, Dal "ritmo" al "canzoniere" cit., p. 424, che elenca una serie di edizioni petrarchesche che portano, fra Otto e Novecento, questo titolo.

4. Ma non si intitolava così la prima edizione di Petrarca a c. di Contini: Francesco Petrarca, Rerum vulgarium fragmenta, con una nota al testo di Gianfranco Contini e indice dei capoversi, Parigi, Tallone, I949; nuova ed., a c. di C. Ossola, 2004. Per un catalogo delle edizioni otto-novecentesche del Canzoniere di Petrarca cfr. Cannata, Dal "ritmo" al "canzoniere" cit., p. 424 e nn. 91 e 92.

5. Confermata anche dal commento Storia e cronistoria del Canzoniere, 1948. Per il Novecento, oltre ai Canzonieri registrati da Gorni, Il canzoniere cit., p. I 20, penso, fra i casi più 'nobili', al Breve canzoniere di Tommaso Landolfi, Firenze, Vallecchi, I97I, e, su un diverso versante d'uso, al Canzoniere italiano: antologia della poesia popolare, a c. di P. P. Pasolini, Milano, Garzanti, 1972. L'impiego del termine è d'altronde vastissimo: a titolo di curiosità registro anche il Canzoniere comunista di Spartacus Picenus, s.l., I954.

6. Se è vera l'identificazione proposta da G. Billanovich, sulla base di una postilla apposta da Petrarca alla sua copia di Orazio: cfr. G. Billanovich, L'Orazio Morgan e gli studi del giovane Petrarca, poi in Id., Petrarca e il primo umanesimo, Padova, Antenore, 1996, pp. 4I- 58 (la postilla si riferirebbe ai primi anni Trenta del Trecento). Ma all'identificazione di Billanovich si è opposto M. Feo, Un "libellus" e gli amanti perpetui (versione riveduta della nota compresa nella sua 'voce' Petrarca di Orazio. Enciclopedia Oraziana cit., p. 423) in Petrarca nel tempo. Tradizione, lettori e immagini delle opere, a c. di M. Feo, Pontedera, Bandecchi e Vivaldi, 2003, pp. 79-80 (ivi anche altri esempi dell'uso petrarchesco di libelluss). Più in generale è utile H. W. Storey, $D i$ libello in libro: problemi materiali nella poetica di Monte Andrea e Dante, in Da Guido Guinizelli a Dante. Nuove prospettive sulla lirica del Duecento, a c. di F. Brugnolo e G. Peron, Padova, Il Poligrafo, 2004, pp. 271-90.

7. Cfr. F. Brugnolo, Il libro di poesia nel Trecento, in Il libro di poesia dal copista al tipografo, a c. di A. Quondam e M. Santagata, Modena, Panini, I989, pp. 9-23, a pp. 9-10. 


\section{ONOMASTICA PETRARCHESCA}

8. Cannata, Dal "ritmo" al "canzoniere" cit., pp. 424 ss., informa sugli usi diversi del termine nella critica di oggi, sottolineandone anche gli eccessi e i fraintendimenti.

9. Il saggio di Avalle non è d'altronde la sola voce importante sulla tipologia del libro di rime antico: dopo V. Bertolucci, Morfologie del testo medievale, Bologna, Il Mulino, I989, pp. 87-I 24, ricordo almeno M. Santagata, Dal sonetto al Canzoniere. Ricerche sulla preistoria e la costituzione di un genere (I 979), Padova, Liviana, $1989^{2}$; S. Longhi, Il tutto e le parti nel sistema del canzoniere, in «Strumenti critici», XIII, 39-40 (I 979), pp. 265-300; F. Brugnolo, Il libro di poesia nel Trecento, in Il libro di poesia dal copista al tipografo cit., pp. 9-23; ancora Santagata, I frammenti dell'anima. Storia e racconto nel Canzoniere di Petrarca, Bologna, Il Mulino, I992, in part. pp. I03-37; e infine il numero monografico di «Critica del testo» su L'antologia poetica, II, I 999.

ı. All'interno di questi Studi, ultimo volume dell'edizione dei Canzonieri antichi, quasi tutti i saggi contengono fin dal titolo ampi riferimenti alla 'forma-canzoniere': una forma che viene ora correttamente fatta retrocedere anche alla letteratura medio-latina e romanza, come ha notato da ultima M. L. Meneghetti, La forma-canzoniere fra tradizione mediolatina e tradiżioni volgari, in L'antologia poetica, in «Critica del testo», II (I999), pp. I I9-40.

I I. Si veda di seguito qualche esempio del vario uso del termine canzoniere nel secondo Novecento: GuitTone D'Arezzo, Canzoniere: $i$ sonetti d'amore del codice Laurenziano, a c. di L. Leonardi, Torino, Einaudi, I 994; F. Brugnolo, Il Canzoniere di Nicolò de’ Rossi, 2 voll., Padova, Antenore, I974-1977; Alessandro SForza, Il Canzoniere, edizione critica e commento a c. di L. Cocito, Milano, Marzorati, I973; G. Gorni, Un canzoniere adespoto di Mariotto Davanzati. Metrica e filologia attributiva, in «Studi di filologia italiana», XXXIII (I975), pp. I89-2 I9; Gasparo Visconti, I canzonieri per Beatrice d'Este e per Bianca Maria Sforza, ed. critica a c. di P. Bongrani, Milano, Fondazione Arnoldo e Alberto Mondadori, "Il Saggiatore", I979; Angelo Galli, Canzoniere, edizione critica a c. di G. Nonni, Presentazione di E. Cecchini, Urbino, Accademia Raffaello, i987; Matteo Maria Boiardo, Canzoniere. Amorum libri, Milano, Garzanti, i 990; Lorenzo DE’ Medici, Canzoniere, a c. di T. Zanato, Firenze, Olschki, I991. Per esprimere una raccolta di poesie sparse, non organiche, è invece molto più diffuso il titolo di Rime, applicato ad esempio, in massa, ai Siciliani, a Guinizelli, a Cavalcanti, a Dante, ai minori del Trecento e del Quattrocento (come nelle antologie di Giuseppe Corsi e di Antonio Lanza).

I 2. Come fa ad esempio Marco Santagata nel corpo della sua edizione: Francesco Petrarca, Canzoniere, edizione commentata a c. di M. Santagata, in Opere italiane, I, Milano, Mondadori, I 996 (nuova ed. 2004). Anche M. Feo, Francesco Petrarca, in Storia della letteratura italiana diretta da E. Malato, vol. X, La tradizione dei testi, Roma, Salerno Editrice, 200 I, pp. 271-329, a p. 277, avverte della necessità di servirsi del nome Canzoniere come di un nome comune.

I 3. E un'altra minuzia grammaticale è quella che preferisce servirsi dell'iniziale minuscola nel primo caso, della maiuscola nel secondo.

I4. Non fa eccezione neppure l'uso di Canzoneri per indicare le rime del Perleone, attestato dalla stampa delle sue rime del 1492 (Napoli, Cantovo), e segnalato da Cannata, Dal "ritmo" al "canzoniere" cit., p. 428. Anche in questo caso il nome non è proprio, ma comune.

I 5. Adotto la distinzione stabilita da G. Genette, Soglie (1987), Torino, Einaudi, I989, pp. 77-79 (a cui rinvio anche per altri aspetti della titolologia).

I6. Dopo Santagata, Dal sonetto al Canzoniere cit., affrontano dai diversi punti di vista il problema, ancora dibattuto, della primogenitura del 'canzoniere' di Guittone nel ms. Laurenziano Rediano 9 M. Picone, Guittone e i due tempi del "Canzoniere", in Guittone d'Arezzo nel settimo centenario della morte, a c. di M. Picone, Firenze, Cesati, I995, pp. 73-88; R. Leporatti, Il 'libro' di Guittone e la "Vita nuova", in «Nuova Rivista di Letteratura Italiana», IV (200I), pp. 4I-I 5o, e L. Leonardi, Guittone nel Laurenziano. Struttura del Canzoniere e tradizione testuale, 


\section{Paola Vecchi Galli}

in La filologia romanza e i codici, Atti Messina, 19-22 dicembre 1991, Messina, Sicania stampa, I993, pp. 443-80, e Il canzoniere Laurenziano: struttura, contenuto e fonti d'una raccolta d'autore, in Studi critici cit., pp. I 53-2 I4

17. F. Rico, "Rime sparse", "Rerum vulgarium fragmenta". Para el titulo y el primer soneto del "Canzoniere", in «Medioevo romanzo», III (1976), pp. IoI-38, poi in Id., Estudios de literatura y otras cosas, Barcelona, Destino, 2002, pp. I I I-46.

I 8. Mi sembra che sia una circostanza testuale (la separazione di una iunctura che altrove Petrarca propone appaiata) mai notata dai commentatori del Canzoniere.

19. La iunctura non compare nella rubrica che Boccaccio trascrive a c. $43 \mathrm{v}$ del Canzoniere Chigiano L.V.r76, dove manca l'aggettivo volgare: «Uiri illustris atque poete celeberrimi francisci petrarce de florentie rome nuper laureati fragmentorum liber incipit feliciter».

20. S. Rizzo, Il Petrarca, il latino e il volgare, in «Quaderni petrarcheschi», VII (1990), pp. 7-40, e Ead., Il latino del Petrarca e il latino dell'umanesimo, in Il Petrarca latino e le origini dell'Umanesimo, in "Quaderni petrarcheschi», IX-X (1992-I 993), vol. I, pp. 349-65; sulla stessa linea Cannata, Dal "ritmo" al "canzoniere" cit., pp. 398-99.

2I. Fanno eccezione i titoli danteschi e quelli di opere in prosa, tuttavia non pertinenti all'ambito lirico: per il quale, si noti, è il manoscritto nel suo insieme a recare un titolo, come nel caso del tardo-duecentesco ms. Vat. Lat. 3793 , Libro de varie romanze volgari. Che la rubrica dei Fragmenta non sia di mano di Petrarca, ma di Malpaghini è detto a chiare lettere da S. Zamponi, Il libro del Canzoniere: modelli, strutture, funzioni, in Rerum vulgarium fragmenta. Codice Vat. Lat. 3195, Commentario all'edizione in facsimile, a c di G. Belloni, F. Brugnolo, H. W. Storey e S. Zamponi, Roma-Padova, Antenore, 2004, pp. 13-72, a p 24.

22. Nel suo intervento (Francesco Petrarca e la fondazione del genere lirico) per il Convegno Internazionale "Petrarca, l’Umanesimo e la civiltà europea", Firenze, 5-Io dicembre 2004.

23. L'intitolazione è a c. 43 v del Chigiano: cfr. Il codice Chigiano L. V.r76 di Giovanni Boccaccio, edizione fototipica, introduzione di D. De Robertis, Roma-Firenze, Alinari, I 976. Si tratta di un titolo raro per il Canzoniere: Cannata, Dal "ritmo" al "canzoniere" cit., p. 4I 8 e n. 77, ne segnala una sola altra occorrenza nota nel ms. di Manchester, John Rylands Library I, anteriore al $\mathrm{I} 383$.

24. Joha. Francisci Suardi Fragmenta vulgaria, a c. di A. Cinquini, Roma, Signorelli, igr 7.

25. Ne ha parlato B. Bentivogli, La poesia in volgare. Appunti sulla tradizione manoscritta, in Bentivolorum Magnificentia. Principe e cultura a Bologna nel Rinascimento, a c. di B. Basile, Roma, Bulzoni, 1984, pp. 177-222.

26. Diversi estratti dei Rerum vulgarium fragmenta di Cosmico sono riprodotti in Rimatori veneti del Quattrocento, a c. di A. Balduino, Padova, Clesp editrice, I980, pp. го4-I 3.

27. Cfr. Domenico da Prato, XX. Prefazione, in Lirici toscani del Quattrocento, a c. di A. Lanza, I, Roma, Bulzoni, 1973, p. 5 I I.

28. Cfr. Cannata, La percęione del Canzoniere cit., p. I64, n. 4.

29. Rinvio perciò per altri dettagli alle due schede che verranno pubblicate in quel Catalogo, mentre del ms. Rediano I 84 parlerò anche più avanti, a testo.

30. Per entrambi i mss. il rinvio è a Signorini, Fortuna del "modello-lirico" Canzoniere cit., pp. I 44-47. 


\section{ONOMASTICA PETRARCHESCA}

31. Aggiungo che nel primo Quattrocento anche la vita di Petrarca di P. Pietro da Castelletto ricorda che Petrarca ha composto fra l'altro un librum rerum vulgarium (cfr. A. Solerti, Le Vite di Dante, Petrarca e Boccaccio scritte fino al secolo decimosesto, Milano, Hoepli, i 904, p. 272).

32. Ora ristampata in ed. anastatica: Francesco Petrarca, Rerum vulgarium fragmenta. Anastatica dell'edizione Valdezoco, a c. di G. Belloni, Padova, I472, Venezia, Regione del Veneto-Marsilio, 200 I.

33. Secondo le tipologie descritte da Signorini, Fortuna del "modello-lirico" Canzoniere cit.

34. Su questo aspetto della produzione e della comunicazione libraria, nel momento cruciale del passaggio dal manoscritto alla tipografia, è illuminante il volume di L. Baldacchini, Aspettando il frontespizio. Pagine bianche, occhietti e colophon nel libro antico, Milano, Edizione Sylvestre Bonnard, 2004, in particolare pp. I I-25.

35. Per questi due manoscritti rinvio rispettivamente a Signorini, Fortuna del "modello-lirico" Canzoniere cit., pp. I48-53, e a M. Feo, "In vetustissimis cedulis". Il testo del postscriptum della senile XIII II gamma e la "forma Malatesta" dei Rerum vulgarium fragmenta, in Verso il Centenario, a c. di L. Chines e P. Vecchi Galli, «Quaderni petrarcheschi», XI (200I), pp. I I 9-48

36. Che Petrarca fosse in altre circostanze ben consapevole del valore unificante del titolo dimostra infatti la sua attenzione alla scelta del nome (del titolo!) anche per altre sue raccolte, come quella delle epistole Familiares (in Fam. I i, commentata da Feo, Fili petrarcheschi cit., pp. I 4 ss.).

37. Non è un titolo contemporaneo la definizione di Canzonero antico che compare sulla costola del miscellaneo ms. Chigiano L.VIII.305: cfr. da ultimo la scheda codicologica di D. De Robertis in Dante Alighieri, Rime, I documenti, I **, Firenze, Le Lettere, 2002, pp. $75^{2-59}$, a p. 755 .

38. I titoli delle raccolte poetiche, in quei casi particolarmente vivaci e accattivanti, vanno di conserva con l'invenzione della stampa (e rappresentano quindi, a differenza del manoscritto, un elemento librario dai chiari intenti promozionali e commerciali): oltre a Gorni, $I l$ Canzoniere cit., altre riflessioni sull'onomastica vengono dall'intervento di Guglielmo Gorni per il Convegno Internazionale "Petrarca, l'Umanesimo e la civiltà europea", Firenze, 5-Io dicembre 2004 (Francesco Petrarca e la fondazione del genere lirico); e cfr. anche N. Cannata, Il canzoniere a stampa, I470-I530: tradizione e fortuna di un genere fra storia del libro e letteratura, Roma, Bagatto, 2000 .

39. Sul Cansonero del Conte di Popoli (fatto oggetto in passato degli studi di Maria Corti e di Marco Santagata) cfr. da ultimo Cannata, Dal "ritmo" al "canzoniere" cit., pp. 427-28.

40. Cannata, La percezione del Canzoniere cit., p. I65, segnala altri due usi del termine Canzoniere applicato a Petrarca in pieno Cinquecento, e cioè nell'edizione Giolito del I $554 \mathrm{e}$ nell'edizione di Castelvetro, Basilea del i 582 . È comunque significativo che il Vocabolario degli Accademici della Crusca, I6r2, non registri ancora il termine canzoniere, così come le Osservazioni sopra il Petrarca di Francesco Alunno (I 550 ).

4I. A mia conoscenza, la Giuntina è segnalata en passant solo da Gorni, Il canzoniere cit., p. I 34 .

42. Ne ha parlato E. Scarpa, "Canzoniere": per la storia di un titolo, in «Studi di filologia italiana», LV (1997), pp. I07-9: e avverto che l'espressione canzoniero di Franciescho Petrarcha è confinata nel colophon dell'edizione Piasi. Emanuela Scarpa, osservando come, nell'incipit, la denominazione dei Fragmenta sia invece Canzoneti, conclude: «Dal quale proprio, forse, nasce la designazione di Canzionero, timidamente relegata dall'editore in fondo al volume, e rimasta isolata per lungo tempo, fino al recupero (se di recupero si tratta, e non piuttosto 


\section{Paola Vecchi Galli}

di un'iniziativa indipendente) nell'edizione bolognese del i 5 I 6» (cfr. Scarpa, "Canzoniere": per la storia di un titolo cit., p. I०8). Si tratta di un'ipotesi discussa e scartata da Cannata, Dal "ritmo" al "canzoniere" cit., pp. 4I I-I 2, e sulla quale nutro anch'io forti perplessità.

43. È una postilla scoperta da Bruno Bentivogli (Il ms. Silvestriano 289 dell'Accademia dei Concordi di Rovigo, in «Studi e problemi di critica testuale», 35, 1987, pp. 27-90, a pp. 36 e 65), che ha sottolineato la difficoltà di datarla esattamente, indicandone però a questo giro di anni la collocazione più probabile.

44. Entrambi i mss. sono segnalati da Cannata, Dal "ritmo" al "canzoniere" cit., pp. 4I 2-I 3 (la cit. da p. 4I 2).

45. Cannata, Dal "ritmo" al "canzoniere" cit., p. 4I I.

46. Cfr. Ricordi sulla vita di Messer Francesco Petrarca e di Madonna Laura scritti da Luigi Peruzzi, Bologna, Romagnoli, I 866, p. 9.

47. Cannata, Dal "ritmo" al "canzoniere" cit., p. 413.

48. Cannata, Dal "ritmo" al "canzoniere" cit., p. 413.

49. È il catalogo - di prossima pubblicazione - che aggiorna quello dei Codici latini del Petrarca nelle Biblioteche fiorentine, a c. di M. Feo, Firenze, Le Lettere, 1991: qui verranno stampate anche le schede codicologiche e critiche di due dei manoscritti sotto citati, il Laurenziano Rediano I 84 e il XC inf. 2, di cui è responsabile chi scrive, con la collaborazione di Stefano Cremonini e di Francesca Siviero.

50. Cfr. De Robertis, in Dante Alighieri, Rime, I documenti, I *, pp. I76-82.

5I. Cfr. I manoscritti datati della Biblioteca Riccardiana di Firenze II, a c. di T. De Robertis e R. Miriello, Firenze, SISMEL-Edizioni del Galluzzo, 1999, pp. 34 e 39.

52. Cfr. in proposito P. Vecchi Galli, Petrarca fra Tre e Quattrocento, in Storia della letteratura italiana diretta da E. Malato, vol. XI, La critica letteraria dal Due al Novecento, coord. P. Orvieto, Roma, Salerno Editrice 2003, pp. I6I-88, in particolare pp. 173 ss.

53. Avverto, fra l'altro, che i due numeri 38 sembrano ritoccati con inchiostro più scuro, forse d'altra mano. La canzone di cui parla la didascalia è in realtà $R v f$. 37 , qui mutila dei primi $60 \mathrm{vv}$.

54. Il Canzoniere di Petrarca trascritto nel ms. Rediano i 84 non segue la forma definitiva (o Vaticana), ma una forma ibridata con la 'Malatesta' e arricchita dall'aggiunta di dodici rime disperse, alcune delle quali malamente attribuite, dei Triumphi adespoti e anepigrafi, della nota obituaria di Laura e di due carmi latini, delle Vite di Dante e Petrarca di Leonardo Bruni, e della Vita di Petrarca di Pier Paolo Vergerio.

55. Su cui cfr. Bandini, V, coll. 95-98.

56. Cfr. Bandini, V, coll. 4I I-4I 2; Codici latini del Petrarca nelle Biblioteche fiorentine cit., pp. I 54-55, n. I00.

57. Cfr. Feo, Fili petrarcheschi cit., p. I 5.

58. Cfr. Cannata, Dal "ritmo" al "canzoniere" cit., pp. 427-28.

59. Segnalato da Cannata, Dal "ritmo" al "canzoniere" cit., p. 427, su indicazione di Giulio Lepschy. 